\title{
IV. A simplified dispersion-photometer
}

\section{Professors W.E. Ayrton \& John Perry}

To cite this article: Professors W.E. Ayrton \& John Perry (1882) IV. A simplified dispersionphotometer, Philosophical Magazine Series 5, 14:85, 45-51, DOI: 10.1080/14786448208628416

To link to this article: http://dx.doi.org/10.1080/14786448208628416

曲 Published online: 28 Apr 2009.

13 Submit your article to this journal 준

Џll Article views: 3

Q View related articles $\asymp$ 
out. On the second day we made it give out energy more rapidly than the first, and on the third more rapidly than on

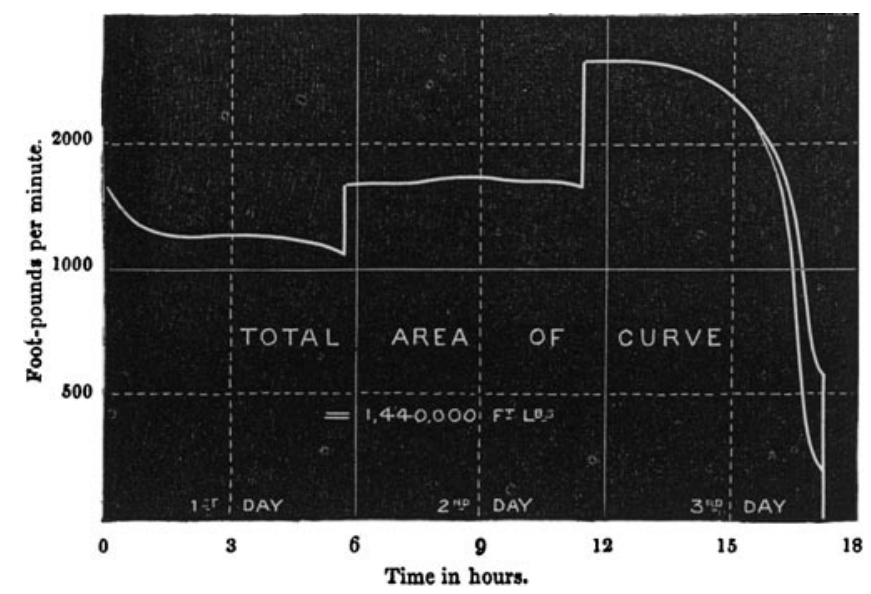

the second, this being done of course by diminishing the total resistance in circuit. During the last day we were discharging with a current of about 25 amperres. And this cell, like the others, showed, on being insulated after having been apparently totally discharged, that there was still a large charge stored up; hence the numbers given above for the capacity are probably under the total value.

Deterioration.-As to deterioration, two months constant charging and discharging of the two accumulators under test showed no signs of deterioration.

IV. A Simplified Dispersion-Photometer. By Professors W. E. Ayrton and John Perry*.

$\mathrm{T}^{\mathrm{T}}$ will be in the recollection of the Members that in 1879 1 we described to the Society a dispersion-photometer which enabled measurements to be made of the intensity of the strongest electric light in a small room and for the rays coming from the electric light at any angle-two essentials which appeared to us necessary in an electric-light photometer. The principle of this photometer consisted in our use of a concave lens to weaken the strength of the light, so as to make the illumination of a screen comparable with the illumination of a standard candle, instead of keeping the lamp a distance of

* Communicated by the Physical Society, having been read at the Meeting on February 25, 1882. 
50 or 100 feet away, which was the plan in use until that time. We exhibit now five successive forms of the instrument, which illustrate the history of its development to the present time.

1. The first of these is very nearly the same as that described in our former paper, with the exception that we discarded the use of a long screw (shown in our original figure) for adjusting the position of the lens-as we found that a very easy adjustment might be effected with the fingers, the tension of the bellows part making an automatic clutch which fixed the lensslide in any position.

2. The second specimen is on the same principle, only that telescope-tubes are used instead of a wooden frame and a bellows. Instead of the lens part alone tilting when the elevated or depressed light has to be examined, the candle-box is here made to tilt also, the candle being supported in gimbals so that it may remain vertical for every angle of elevation.

3. The third specimen is on pretty much the same principle; but as we found a difficulty in comparing two illuminated disks whose centres were some distance apart, we arranged in front of these disks two mirrors, which enable us to make the comparison between two illuminated semicircles having the same diameter. The difficulty of adjusting the lens and making a comparison of the illuminations, and reading the scale, without moving one's head, in all these early instruments led us to the

4th form, which is probably familiar to the Members, as it was exhibited at Paris and largely used there for measurements. In this the candle-box and the lens-box are placed end to end, the lens is fixed in a wooden piston which moves in its hollow square box, which is lined with velvet; and the lens shows its position by a pointer moving over the scale outside. The pointer projects from the inside of the wooden cylinder at any point of a long slot, whose sides are made of india-rubber tubing, so that no extraneous light can reach the illuminated screen. A little handle working a rack and pinion enables the lens to be placed in any position. Through a hole at the side the two screens can be viewed reflected in two mirrors, inclined to one another in the space between the candle-box and the lens-cylinder; and the illuminated papers are viewed as two semicircles having a common diameter. In front of this hole we have slides of red and green glass ; so that, as our custom has always been, we make two measurementsone a comparison of the ruby-red light of the lamp examined with the red light of the candle, and another of the green lights. This instrument differed from the earlier forms in not requiring any calculation to be made of the strength of the 
light; that is, the reading of the pointer was not merely a reading of its distance from the screen, but it was a reading in standard candles of the power of the light. Three such scales were placed on the instrument; and there were three certain distances at which the lamp had to be placed for examination. The tilting-arrangement was of course different from that of the earlier forms.

As the instrument had by this time (the end of last year) come into a rather extensive practical use, we had opportunities of seeing that, as an instrument to bo used by unscientific persons, it was not yet in a perfect condition, in spite of the many changes that had been made in its construction. The most important difficulty was due to the fact that a slight lateral change in the position of the observer's eye caused the apparent illumination of the screens to vary. Being aware of this fact ourselves, we maintained a certain fixed position of the eye when making observations; but the instrument could not at once be used by persons not accustomed to make delicate experiments.

5. The fifth form, which we now present to the Society, is the outcome of our labours on this subject. We have all along seen the disadvantage of using the Bouguer's twoscreen method, since, when lights are examined that have passed through tissue or tracing-paper, a very slight change in the position of the observer's eye makes a very great difference in the apparent illumination, whereas, using Rumford's method, when a sheet of white blotting-paper is employed as a screen very considerable changes in the position of the eye produce no change in the apparent illumination-a result, however, which is not attainable when ordinary drawing-paper is used as the screen. If, however, Rumford's method is to be used to measure the rays coming at different angles from an electric light, a mirror must be employed to reflect them successively onto the same screen; and if used in the ordinary way, the angle of incidence of the rays on the mirror will be different in different cases. Now the difficulty that always met us arose from the inequality of the reflecting-power of an ordinary mirror for rays falling on it at different angles of incidence. We have, however, completely overcome this difficulty in an extremely simple way, by causing the mirror to turn about a horizontal axis inclined at $45^{\circ}$ to its plane, and the whole photometer to turn about a vertical axis. With this arrangement the angle of incidence, and consequently the proportional absorption, is the same whatever be the inclination of the rays coming from the lamp to the mirror; and, further, the angle being $45^{\circ}$, the amount of rotation of the 
mirror about its horizontal axis necessary to enable measurements to be made of rays coming at any angle, after measurements have been made of the horizontal beam, is exactly equal to the inclination of the beam in question.

Using Rumford's method in this latest form of our photometer, we are to a great extent indejondent of the presence of other sources of illumination of the screen, so that the apparatus need not be enclosed in a box. At the same time, however, the sensibility of the test is much increased by placing a shade to prevent the electric light shining directly onto the screen. On this screen of blotting-paper, $B$, is thrown the shadow of a black rod, $A$, placed in front of it, by a candle in the candle-holder, $\mathrm{D}$.

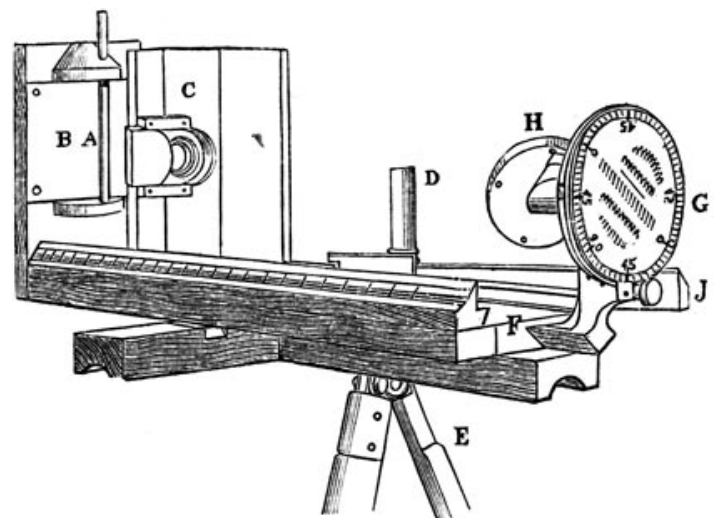

Now it is well known that if an electric light is also allowed to illuminate this screen, and to throw a second shadow of the $\operatorname{rod} \mathrm{A}$ on the paper, and if the candle is adjusted at such a distance that the two shadows are of equal intensity, the strength of the light is to that of the candle in the ratio of the squares of their distances from their respective shadows. But instead of allowing the strong light to pass directly to the screen, we cause it to pass through the concave lens in the sliding wooden frame C. A pointer on this slide tells the distance of the lens from the screen. As you are all aware, the weakening of light-intensity produced by the lens enables us to leave our electric lamp within a few feet of the instrument. We have experimentally found that there is no appreciable loss of light in passing through the lens. The candle slides on the bar $\mathrm{J}$; and its distance from its shadow is shown by a pointer on a scale. If $f$ is the focal length of the lens, $D$ the distance of the electric light from the paper-screen, $d$ 
the distance of the centre of the lens from the screen, and $c$ that of the candle when the shadows show equal illumination, then, if $\mathrm{L}$ is the strength of the examined source of light in standard candles,

$$
\sqrt{\mathrm{L}}=\frac{\mathrm{D}-d}{c}\left\{1+d\left(\frac{1}{f}+\frac{1}{\mathrm{D}-\bar{d}}\right)\right\}
$$

or

$$
\mathrm{L}=\frac{1}{c^{2}}\left\{\mathrm{D}+\frac{d(\mathrm{D}-d)}{f^{2}}\right\}^{2}
$$

For our own use we prefer to employ the formula; but as all the common instruments which have hitherto been manufactured have lenses whose focal length is 4 inches, we have prepared a table, a copy of which is sent out along with each instrument, in which the value of $\mathrm{L}$ is given for various values of $\mathrm{D}, d$, and c. Using this table, it is necessary to have the lamp at either 60,120 , or 300 inches from the screen; the candle is either at $10,14 \cdot 14$, or 20 inches from the screen; and the table is made out for every half inch of the lens-scale. But inasmuch as we find that the improved arrangement of the mirror already referred to constitutes perhaps the most useful part of the instrument, and as the use of this improvement involves many alterations of $\mathrm{D}$, the manufacturer proposes in future not to furnish any table of values of $L$ unless specially asked for.

$\mathrm{H}$ is the plane silvered-glass mirror which makes the angle of $45^{\circ}$ with the axis of the lens, and with the axis about which the mirror itself is free to revolve. As already explained, a ray of light reflected from the mirror and passing through the centre of the lens must, for any position of the mirror, have an angle of incidence of $45^{\circ}$, and so must experience the same amount of absorption, from whatever direction it may have come to the mirror. Further, this angle being $45^{\circ}$, a fixed pointer marks on the graduated circle $G$, which moves with the mirror, the angle which any ray we may be examining makes with the horizontal.

In this instrument we find that from 30 to 34 per cent. of the incident light at $45^{\circ}$ is absorbed, whether this light is of ruby-red or signal-green colour; so that we have the easy practical rule for all cases-add one half to the measured intensity of light reflected.

We need not here refer to the fact that, when investigating the efficiency of an electric lamp, we always measure the horse-power given electrically to the lamp simultaneously with the photometric measurement.

The lamp is suspended in such a way that it can readily be Plil. Mag. S. 5. Vol. 14. No. 85. July 1882 . E 
placed at any elevation. The frame of the tripod-stand is first levelled. A pin at F, directly underneath the centre of the

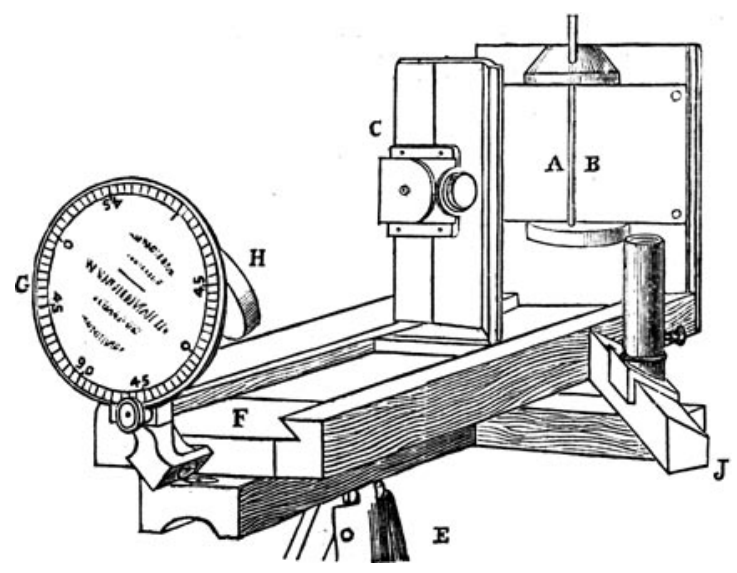

mirror, passes through the base of the photometer and fits into a hole in the top of the tripod-stand. The photometer, by turning round this pin, can, without producing any change in the distance of the centre of the mirror from the lamp, and therefore without changing the distance from the screen to the lamp, receive the small horizontal motion necessary for the adjustment of a new inclination of the rays coming from the electric light, without any alteration of the distance of the centre of the mirror from the lamp. The divided circle is clamped with the index at $0^{\circ}$; the lamp is lowered or raised till the illuninated disk formed by the reflected light, passing afterwards through the lens, is in the middle of the paper screen. A little sliding shutter with a fine hole in its centre, seen in the figure, enables a very exact adjustment to be made; but in practice we find that we get sufficient accuracy without the use of the shutter. We now measure the distance from lamp to centre of mirror in inches. Equalizing the intensities of the two shadows by adjusting the lens-slide when looking at them through red or green glass, we now note the lens- and candle-readings; and we repeat these operations, changing from red to green and green to red about five times in a minute. The lamp is now raised or lowered and fixed in any position; a few seconds suffice to turn the mirror so that it sends its centre ray exactly through the centre of the lens. The distance from screen to mirror in this instrument being 22 inches, if $\delta$ is the distance from centre of mirror to vertical from lamp. 
and if $\theta$ is the angle of elevation, then

$$
\mathrm{D}=22+\delta \sec \theta \text {. }
$$

Using this value of $\mathrm{D}$ in the formula above, and adding one half to the strength of the light to make up for absorption, the true intensity of the light in standard candles can be ascertained. We find in practice that, if an electric light is moderately steady, ten measurements may be made, with some confidence in their accuracy, in two minutes; and the light may be measured in ten different positions, from an angle of depression of $60^{\circ}$ to an angle of elevation of $60^{\circ}, 100$ observations being taken, in less than half an hour.

We may mention one very important result we have been led to by the systematic employment of a photometer which can be used close to the electric light; and that is the large amount of absorption that occurs on certain days when the rays fiom strong electric lights, and especially the green rays, pass through the air which appears to the eye perfectly clear. At first we were inclined to think the higher results for the candle-power of a lamp obtained with our dispersion-photometer than those obtained with an ordinary distance-photometer were due to some error in our photometer itself; but we have since ascertained that this is due to the absorption of the air-because we find that, if simultaneous measurements are macle with ordinary Rumford's photometers, each witbout lens or mirror, placed at different distances from the limp in the same azimuth and in the same horizontal plane, the nearer one gives, as a rule, the highest readings; and the difference is the greater the stronger the light, and is greater if the light be examined at each photometer with green glass.

V. On the Connexion between Viscosity and Density in Fluids, especially Gaseous Fluids. By E. Warburt and L. v. BABO*.

THE laws according to which the elasticity and viscosity of a body are comnected with its density are of great simplicity in the case of gaseous bodies. The elasticity of these, $i$. e. the reciprocal of their compressibility, is given, according to Boyle and Mariotte's law, by the pressure, and is proportional to the density; the viscosity, measured by the coefficient of friction, is, according to Maxwell's law, independent of the density.

It is known that the first of these laws, that which refers to

* Translated from the Sitzungsberichte der K. Preuss. Akadenie der Wissenschaften $\approx$ Berlin, May 4, 1882, pp. 509-514. 